\title{
Spectral modelling of gamma-ray blazars
}

\author{
M. Böttcher, H. Mause and R. Schlickeiser \\ Max-Planck-Institut für Radioastronomie, Postfach 20 24, D - 53010 Bonn, Germany
}

\begin{abstract}
We present model calculations reproducing broadband spectra of $\gamma$-ray blazars by a relativistic leptonic jet, combining the EIC and the SSC model. To this end, the evolution of the particle distribution functions inside a relativistic pair jet and of the resulting photon spectra is investigated. Inverse-Compton scattering of both external (EIC) as well as synchrotron photons (SSC) is treated using the full KleinNishina cross-section and the full angle-dependence of the external photon source. We present model fits to the broadband spectra of Mrk 421 and 3C279 and the X-ray and $\gamma$-ray spectrum of PKS 1622-297. We find that the most plausible way to explain both the quiescent and the flaring states of these objects consists of a model where EIC and SSC dominate the observed spectrum in different frequency bands. For both Mrk 421 and $3 \mathrm{C} 279$ the flaring states can be reproduced by a harder spectrum of the injected pairs.
\end{abstract}

\section{INTRODUCTION}

The discovery of many blazar-type AGNs (e. g., Hartman et al. 1992) as sources of high-energy $\gamma$-ray radiation, dominating the apparent luminosity, has revealed that the formation of relativistic jets and the acceleration of energetic charged particles, that generate nonthermal radiation, are key processes to understand the energy conversion process. Emission from relativistically moving sources is required to overcome $\gamma$-ray transparency problems implied by the measured large luminosities and short time variabilities (for review see Dermer \& Gehrels 1995).

Dermer, Miller \& Li (1996) have recently inspected the acceleration of energetic electrons and protons in the central AGN plasma by comparing the time scales for stochastic acceleration with the relevant energy loss time scales. Their results demonstrate that with reasonable central AGN plasma parameter values lowfrequency turbulence can energize protons to $\mathrm{TeV}$ and $\mathrm{PeV}$ energies where photopair and photo-pion production are effective in halting the acceleration (Sikora et al. 1987, Mannheim \& Biermann 1992). Once the accelerated protons reach the thresholds for the latter processes they will generate plenty of secondary electrons and positrons of ultrahigh energy which are now injected at high energies into the acceleration scheme. The further fate of the secondary particles depends strongly on whether they find themselves in a compact environment set up by the exter- 
nal accretion disk, or not. Secondary particles within the photosphere will initiate a rapid electromagnetic cascade which has been studied by e.g. Mastichiadis \& Kirk (1995), which might even lead to runaway pair production and associated strong X-ray flares (Kirk \& Mastichiadis 1992), and/or due to the violent effect of a pair catastrophy (Henri, Pelletier \& Roland 1993) ultimately lead to an explosive event and the emergence of a relativistically moving component filled with energetic electron-positron pairs.

It is the purpose of the present investigation to follow the time evolution of the relativistic electrons and positrons as the emerging relativistic blob moves out. We generalize the approach used in earlier work (Dermer \& Schlickeiser 1993, Dermer, Sturner \& Schlickeiser 1996) by accounting for Klein-Nishina effects and including external inverse-Compton scattering as well as synchrotron self-Compton scattering self-consistently.

\section{SIMULATIONS OF THE TEMPORAL EVOLUTION OF PARTICLE AND PHOTON SPECTRA}

We assume the relativistic pairs to be isotropically distributed in a spherical volume of radius $R_{B}$, located at height $z_{i}$ above the accretion disk plane. The minimum Lorentz factor of the pairs at the time of injection is expected to be in the range of the threshold value of the primary protons' Lorentz factor for photo-pair production. We find this threshold at $\gamma_{1 \pm} \sim 10^{3}$. The pair distribution above this cutoff basically reflects the acceleration spectrum of the protons which can extend up to $\gamma_{2 \pm} \sim 10^{6}$. The lack of a significant cut-off at high photon energies due to $\gamma-\gamma$ pair production in the EGRET spectra of many $\gamma$-ray blazars and in particular the detection of $\mathrm{TeV} \gamma$-rays from Mrk 421 suggests that a new jet component must be produced/accelerated outisde the $\gamma$-ray photosphere for $\mathrm{TeV}$ photons. We find this photosphere due to the interaction of $\gamma$-rays with accretion disk radiation to be located around $z \approx 5 \cdot 10^{-3} M_{8}^{-1}$ pc where $M_{8}$ is the mass of the central black hole in units of $10^{8} M_{\odot}$.

The blob is assumed to move outward perpendicularly to the accretion disk plane

with velocity $c \beta_{\Gamma}=c \sqrt{1-1 / \Gamma^{2}}$. The cooling mechanisms we take into account are inverse-Compton scattering of accretion disk photons (EIC), synchrotron and synchrotron-self-Compton (SSC) losses to arbitrarily high order. For comparison to observations, we calculate the time-averaged emission, since the integration times of present-day $\gamma$-ray observing instruments are much longer than the cooling timescales resulting from our simulations.

\section{MODEL CALCULATIONS}

We used our code to fit the spectra of three $\gamma$-ray blazars, namely the flatspectrum radio quasar 3C279, the BL-Lac object Mrk 421 and the quasar PKS 1622- 


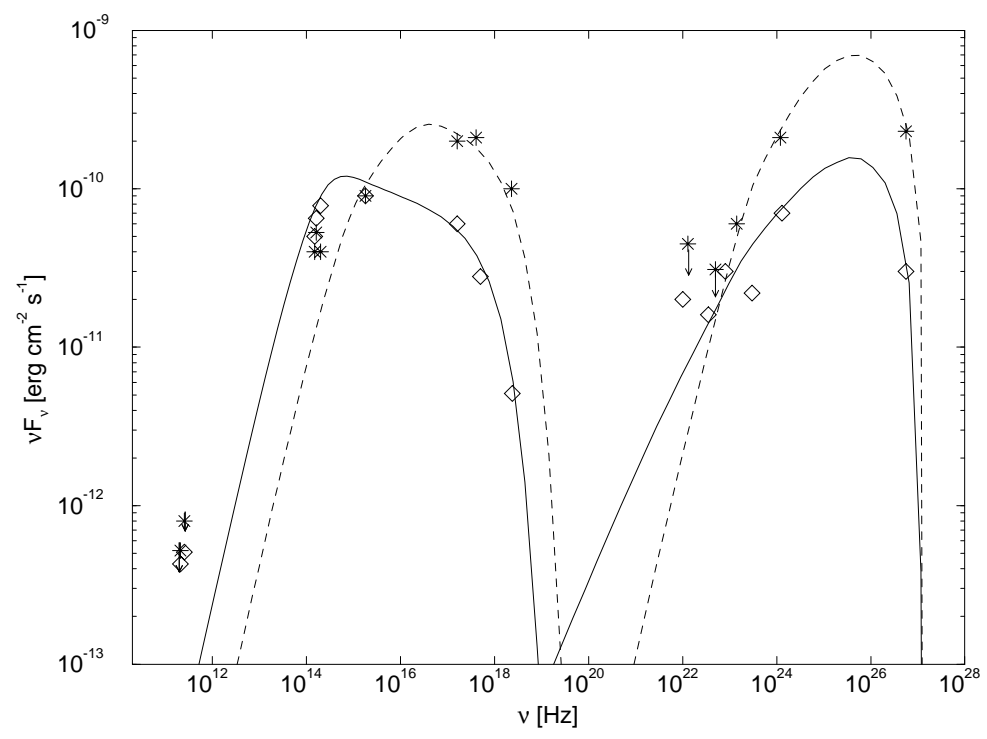

FIGURE 1. Fit to the broadband spectrum of Mrk 421 in its quiescent state (squares; solid line) and during the May 1994 flare (stars; dashed line)

297. Throughout our calculations, we assumed $H_{0}=75 \mathrm{~km} \mathrm{~s}^{-1} \mathrm{Mpc}^{-1}, q_{0}=0.5$, and $\Lambda=0$. The fits to Mrk 421, as shown in Fig. 1, are discussed in detail in Böttcher et al. (1997).

\section{The quasar $3 \mathrm{C} 279$}

The quasar 3C279 was observed in a broad-band campaign by Hartman et al. (1996) during an outburst in 1991 June and in a more quiescent state in 1991 September - October. For the latter phase, unfortunately, no simultaneous observations in the infrared - optical - UV band were available. The typical flare timescale of this object is several days, which restricts the size of the emitting region to $R_{B} \lesssim D \cdot 10^{17} \mathrm{~cm}$ where $D=\left(\Gamma\left[1-\beta_{\Gamma} \cos \theta_{o b s}\right]\right)^{-1}$ is the Doppler factor. Our fits to the quiescent and the flaring phase of $3 \mathrm{C} 279$ are shown in Fig. 2. The parameters for the quiescent state were

$$
\begin{array}{rlrl}
\gamma_{1 \pm} & =200 & \gamma_{2 \pm} & =7 \cdot 10^{4} \\
s & =2.5 & n & =3 \mathrm{~cm}^{-3} \\
R_{B} & =3 \cdot 10^{17} \mathrm{~cm} & B & =0.1 \mathrm{G} \\
L & =10^{46} \mathrm{erg} \mathrm{s}^{-1} & M & =5 \cdot 10^{8} M_{\odot} \\
z_{i} & =6 \cdot 10^{-2} \mathrm{pc} & \Gamma & =15 \\
\theta_{\text {obs }} & =2^{0} & &
\end{array}
$$

where $\gamma_{1,2 \pm}$ are the cutoffs of the initial pair distributions, $s$ is the initial spectral index of the particle spectra $\left(n(\gamma) \propto \gamma^{-s}\right), z_{i}$ is the height of the injection site 


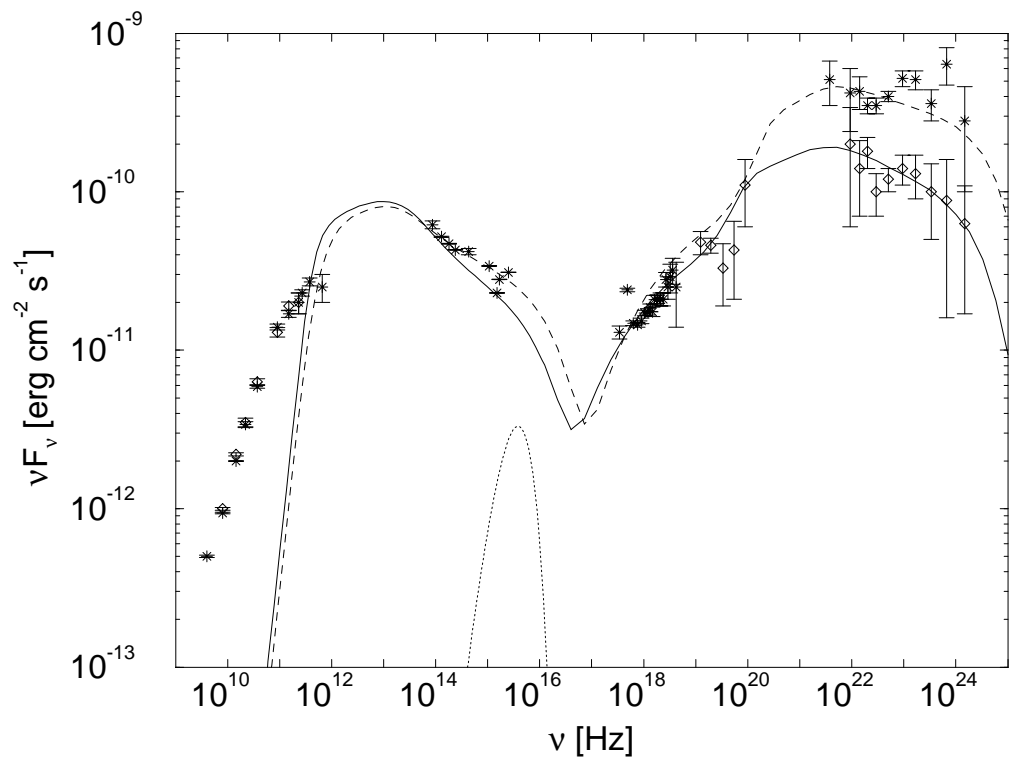

FIGURE 2. Fit to the broadband spectrum of 3C279 in its quiescent state (squares; solid line) during 1991 September - October, and during the flare of 1991 June (stars; dashed line). Dotted curve: accretion disk spectrum

above the accretion disk plane, $L$ is the total luminosity of the accretion disk and $M$ is the masss of the central black hole.

The flaring state could be reproduced by a harder injection spectrum with $s=$ 2.4, $\gamma_{1 \pm}=300$ and $\gamma_{2 \pm}=8 \cdot 10^{4}$.

\section{The quasar PKS 1622-297}

Recently, Mattox et al. (1997) have reported an intense $\gamma$-ray flare of the quasar PKS 1622-297 during which the X-ray and the $\gamma$-ray spectrum can be represented by two different power-laws. Our model calculation, which is illustrated in Fig. 3, demonstrates that the combined SSC/EIC model is well suited to reproduce such a two-component spectrum.

The parameters chosen for the fit shown in Fig. 3 are:

$$
\begin{array}{rlrl}
\gamma_{1 \pm} & =400 & \gamma_{2 \pm} & =7 \cdot 10^{4} \\
s & =2.7 & n & =420 \mathrm{~cm}^{-3} \\
R_{B} & =10^{17} \mathrm{~cm} & B & =0.08 \mathrm{G} \\
L & =10^{46} \mathrm{erg} \mathrm{s}^{-1} & M & =10^{8} M_{\odot} \\
z_{i} & =2.5 \cdot 10^{-2} \mathrm{pc} & \Gamma & =15 \\
\theta_{\text {obs }} & =4^{0} & &
\end{array}
$$




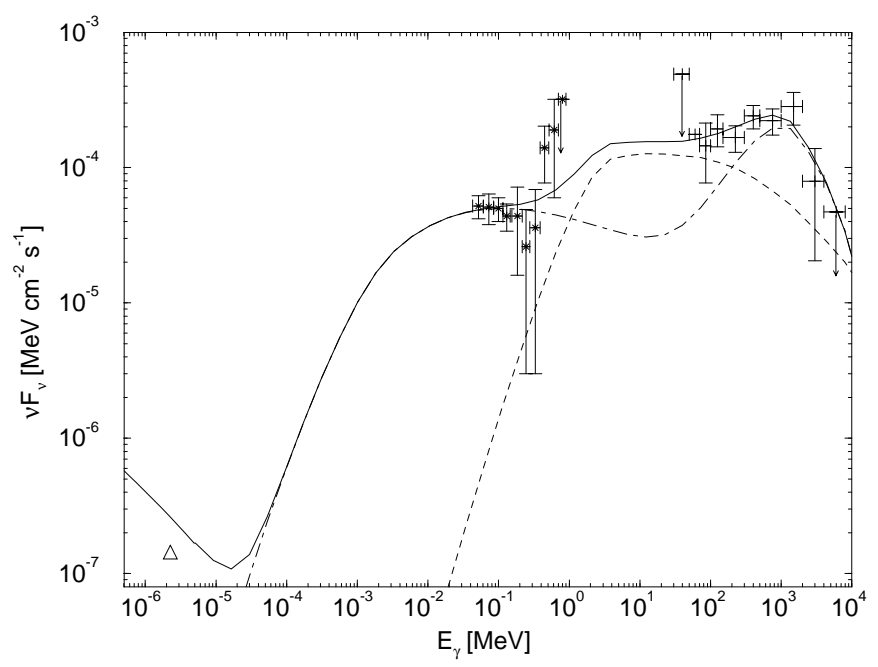

FIGURE 3. Fit to the X-ray and $\gamma$-ray spectrum of PKS 1622-297 during the flare reported by Mattox et al. (1997); the optical data point is from non-simultaneous observations and does not correspond to the flare state of PKS 1622-297. Dashed: EIC, dot-dashed: SSC

\section{REFERENCES}

1. Böttcher, M., Mause, H., \& Schlickeiser, R., A $\mathscr{\xi} A$, in press (1997)

2. Dermer, C. D., and Gehrels, N., ApJ 447, 103 (1995)

3. Dermer, C. D., Miller, J. A., and Li, H., ApJ 456, 106 (1996)

4. Dermer, C. D., \& Schlickeiser, R., ApJ 416, 458 (1993)

5. Dermer, C. D., Sturner, S. J., \& Schlickeiser, R., ApJS, 109, 103 (1997)

6. Hartman, D., et al., ApJ 385, L1 (1992)

7. Hartman, R. C., Webb, J. R., Marscher, A. P., et al., ApJ 461, 698 (1996)

8. Henri, G., Pelletier, G. \& Roland, J., ApJ 404, L41 (1993)

9. Kirk, J. G., \& Mastichiadis, A., Nature 360, 135 (1992)

10. Mannheim, K., \& Biermann, P. L., A\&A 253, L21 (1992)

11. Mastichiadis, A., \& Kirk, J. G., A\&A 295, 613 (1995)

12. Mattox, J. R., Wagner, S. J., Malkan, M., et al., ApJ 476, in press (1997)

13. Sikora, M., Kirk, J., T., Begelman, M. C., et al., ApJ 320, L81 (1987) 